\title{
Supervised exercise therapy versus usual care for patellofemoral pain syndrome: an open label randomised controlled trial
}

\author{
R van Linschoten, sports physician, ${ }^{1}$ M van Middelkoop, researcher, ${ }^{1}$ M Y Berger, researcher, general \\ practitioner, ${ }^{1}$ E M Heintjes, research associate, ${ }^{4}$ I A N Verhaar, professor of orthopaedics, ${ }^{2}$ S P Willemsen, \\ statistician, ${ }^{1,3}$ B W Koes, research professor, ${ }^{1}$ S M Bierma-Zeinstra, associate research professor ${ }^{1}$
}

${ }^{1}$ Department of General Practice, Erasmus University Medical Centre, Rotterdam, Netherlands

${ }^{2}$ Department of Orthopaedics, Erasmus University Medical

Centre

${ }^{3}$ Department of Epidemiology and Biostatistics, Erasmus University Medical Centre

${ }^{4}$ PHARMO Institute, Utrecht, Netherlands

Correspondence to: $\mathrm{R}$ van

Linschoten

r.v.linschoten@hccnet.nl

Cite this as: BMJ 2009;339:b4074 doi:10.1136/bmj.b4074

\section{ABSTRACT}

Objective To assess the effectiveness of supervised exercise therapy compared with usual care with respect to recovery, pain, and function in patients with patellofemoral pain syndrome.

Design Open label randomised controlled trial.

Setting General practice and sport physician practice.

Participants Patients with a new episode of

patellofemoral pain syndrome recruited by their general practitioner or sport physician.

Interventions The intervention group received a standardised exercise programme for 6 weeks tailored to individual performance and supervised by a physical therapist, and were instructed to practise the tailored exercises at home for 3 months. The control group were assigned usual care, which comprised a "wait and see" approach of rest during periods of pain and refraining from pain provoking activities. Both the intervention group and the control group received written information about patellofemoral pain syndrome and general instructions for home exercises.

Main outcome measures The primary outcomes were self reported recovery (7 point Likert scale), pain at rest and pain on activity (0-10 point numerical rating scale), and function (0-100 point Kujala patellofemoral score) at 3 months and 12 months follow-up.

Results A total of 131 participants were included in the study: 65 in the intervention group and 66 in the control group. After 3 months, the intervention group showed better outcomes than the control group with regard to pain at rest (adjusted difference $-1.07,95 \%$ confidence interval -1.92 to -0.22 ; effect size 0.47 ), pain on activity $(-1.00,-1.91$ to $-0.08 ; 0.45)$, and function $(4.92,0.14$ to $9.72 ; 0.34)$. At 12 months, the intervention group continued to show better outcomes than the control group with regard to pain (adjusted difference in pain at rest $-1.29,-2.16$ to -0.42 ; effect size 0.56 ; pain on activity $-1.19,-2.22$ to -0.16 ; effect size 0.54$)$, but not function (4.52, -0.73 to 9.76). A higher proportion of patients in the exercise group than in the control group reported recovery $(41.9 \% \vee 35.0 \%$ at 3 months and $62.1 \%$ $v 50.8 \%$ at 12 months), although the differences in self reported recovery between the two groups were not statistically significant. Predefined subgroup analyses revealed that patients recruited by sport physicians $(n=30)$ did not benefit from the intervention, whereas those recruited by general practitioners $(n=101)$ showed significant and clinically relevant differences in pain and function in favour of the intervention group.

Conclusion Supervised exercise therapy resulted in less pain and better function at short term and long term follow-up compared with usual care in patients with patellofemoral pain syndrome in general practice. Exercise therapy did not produce a significant difference in the rate of self reported recovery.

Trial registration ISRCTN83938749.

\section{INTRODUCTION}

Patellofemoral pain syndrome can be defined as pain around the patella that occurs during or after high loaded flexion and extension of the knee. ${ }^{1-3}$ The main symptom is pain, and the condition generally progresses to impaired function. Physicians, especially general practitioners (GPs) and sport physicians, are frequently confronted with patients who have patellofemoral pain syndrome. Although there are no precise epidemiological data, an average GP practice in the Netherlands reports about five or six new cases a year. ${ }^{4}$ In sport medicine practices, patellofemoral pain syndrome comprises up to $25 \%$ of all new running injuries. ${ }^{56}$ Women are more likely to be affected than men. ${ }^{7}$ Pain usually starts during adolescence when participation in sporting activities is high, ${ }^{7}$ although symptoms can occur over a prolonged period of time. ${ }^{8}$ Extensive diagnostic investigations do not yield specific pathology.

There is no agreement concerning the aetiology of patellofemoral pain syndrome or the most appropriate treatment. There is, however, general consensus that the preferred treatment approach is non-surgical. Rest during periods of pain and refraining from pain provoking activities are advised; this "wait and see" approach is advocated in the Dutch national GP guidelines and is considered usual care. ${ }^{9}$ 
An active approach to treating patellofemoral pain syndrome has been advocated since the 1990s. Strategies range from simple quadriceps strengthening to more complex exercise therapy including taping or bracing. ${ }^{13681011}$ According to a systematic review on the benefits of exercise therapy for patellofemoral pain syndrome,${ }^{12}$ there is only limited evidence that exercise is more effective than no exercise with respect to pain reduction. Furthermore, the evidence as to whether exercise provides functional improvement is conflicting.

The objective of the present study was to investigate in the short term as well as in the long term the effects of exercise therapy compared with usual care in patients with patellofemoral pain syndrome.

\section{METHODS}

The study protocol has been published previously. ${ }^{13}$ Briefly, patients aged between 14 and 40 years consulting their GP or sport physician for patellofemoral pain syndrome were eligible for this trial. Inclusion criteria comprised the presence of at least three of the following symptoms: pain when walking up or down stairs; pain when squatting; pain when running; pain when cycling; pain when sitting with knees flexed for a prolonged period of time; grinding of the patella; and a positive clinical patellar test (such as Clarke's test or patellar femoral grinding test). ${ }^{1415}$ Symptoms had to have persisted for longer than 2 months but not longer than 2 years. Patients were excluded if they had knee osteoarthritis, patellar tendinopathy, Osgood-Schlatter disease, or other defined pathological conditions of the knee, or had previous knee injuries or surgery. Patients were also excluded if they had already been treated with supervised exercise therapy.

Recruitment took place in 38 "HONEUR" practices - general practices allied with the Department of General Practice at Erasmus University Medical Centreand in four sports medical centres in Rotterdam, Leidschendam, Breda, and Gorinchem. Eligible patients were informed about the study and introduced to the research team, who provided patients with more extensive information, checked that patients met the inclusion and exclusion criteria, and obtained informed consent.

After inclusion, patients were randomly allocated to the intervention (exercise therapy) or the control (usual care). The randomisation was done by an independent researcher who used a computer generated list in which patients were stratified by age (14-17 years or 18 years and older) and by recruiting physician (GP or sport physician). A block size of eight was used within the four strata.

\section{Interventions}

Patients in the intervention group followed a standardised exercise protocol tailored to individual achievement and were supervised by a physical therapist. The programme consisted of a general warm up on a bicycle ergometer followed by static and dynamic muscular exercises for the quadriceps, adductor, and gluteal muscles. The programme also included balance exercises and flexibility exercises for major thigh muscles. Patients exercised for 25 minutes supervised by the physical therapist. The load of the exercise programme was increased every 2 weeks during the first 6 weeks by increasing the number of repetitions or the intensity of the exercises.

The increment of the exercise protocol was monitored by the physical therapist who was guided by pain reaction on exertion. Patients visited the therapist nine times in 6 weeks. In addition, they were instructed to practise the exercises daily for 25 minutes over a period of 3 months. To enhance compliance, patients received a tutorial with photographs, a text explaining the exercises, and a diary to register the amount of exercising.

Both the intervention group and the control group received standardised information and advice from their GP or sport physician about the background of patellofemoral pain syndrome and its good prognosis, as well as advice to refrain from all sports activities that provoke pain. Patients were recommended to use a simple analgesic such as paracetamol when pain was severe and to find alternative ways to keep in shape. Instructions for daily isometric quadriceps contractions were given to both groups according to the guidelines for Dutch GPs. ${ }^{9}$ All this information was compiled to a leaflet that was handed to the patients in both groups to promote standardisation (see web extra).

Other interventions - like the use of bandages or braces, insoles, or ice applications, or consumption of medication other than simple analgesics - were allowed in both groups. Information about these additional interventions was collected after 6 weeks and at 3, 6, 9, and 12 months using self report questionnaires. Physicians were instructed not to refer patients in the control group to a physical therapist during the first 3 months of follow-up (that is, when participants in the intervention group were receiving the exercise therapy), and patients in the control group were instructed not to visit a physical therapist during this period.

\section{Outcome measurement}

Follow-up self report questionnaires were filled in by patients at baseline, at 6 weeks, and at 3 months, 6 months, 9 months, and 12 months after inclusion in the study. Primary outcomes measured at 3 and 12 months follow-up were: perceived recovery compared with at the start of the study, measured on a 7 point Likert scale ranging from "completely recovered" to "worse than ever"; functional disability, measured using the Kujala Patellofemoral Scale, a disease specific validated disability scale ranging from 0 (complete disability) to 100 (fully functional) ${ }^{16}$; and pain severity at rest and on activity, measured using a numerical rating scale ranging from 0 (no pain) to 10 (unbearable pain). ${ }^{1718}$

Patients were deemed to have recovered if they rated themselves as "fully recovered" or "strongly recovered" on the Likert scale, whereas those who rated themselves as "slightly recovered" to "worse than 
ever" were deemed to have not recovered. This threshold was used to dichotomise perceived recovery into two clear categories: "recovered" and "not recovered."

\section{Sample size}

Our sample size calculation was based on a previous study by Clark et al that undertook a similar comparison of interventions. ${ }^{19}$ They reported an absolute increase in recovery after one year of $22 \%$ in the exercise therapy group $(19 \%$ recovery in the usual care group compared with $41 \%$ recovery in the exercise therapy group). Such a difference can be detected statistically with 61 patients in each group (power 0.80 , alpha 0.05 , one sided test for the additional value of supervised exercise therapy). We anticipated that we would need a study population of 136 patients, allowing for a potential dropout rate of $10 \%$.

Excluded $(n=32)$ :

Did not meet inclusion criteria $(n=16)$

No final informed consent $(n=10)$

Symptoms diminished $(n=6)$

Patients randomly assigned $(n=131)$

Exercise therapy $(\mathrm{n}=65)$
Didated protocol $(\mathrm{n}=6)$
Additional interventions used (3 months):
NSAIDs $(\mathrm{n}=4)$
Topical agents $(\mathrm{n}=2)$
Bandages/braces $(\mathrm{n}=13)$
Insoles $(\mathrm{n}=9)$
Physical therapy $\left(\mathrm{n}=0^{\#}\right)$

Lost to follow-up at 3 months $(n=2)$ :

Lacked motivation ( $n=1$ at 6 weeks) Unreachable ( $n=1$ at 3 months)

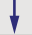

Analysed at 3 months

Analysed for recovery $(n=64)$

Analysed for function and for pain $(n=65)$

\section{t}

Additional interventions used (12 months):

NSAIDs $(n=2)$

Topical agents $(n=2)$

Bandages/braces $(n=5)$

Insoles $(\mathrm{n}=0)$

Physical therapy $(n=13 \#)$

Lost to follow-up at 12 months $(n=5)$ Poor communication/unreachable

$\longrightarrow \quad(n=2$ at 6 months $)$ Unreachable ( $\mathrm{n}=1$ at 9 months and $\mathrm{n}=2$ at 12 months)

At 12 months

Analysed for recovery $(n=64)$

Analysed for function and for pain $(n=65)$

Fig 1 | Flow chart of the recruitment, inclusion, assignment, and subsequent follow-up of the study patients. \#=physical therapy additional to intervention

\section{Statistical analysis}

Differences between the intervention and control group were analysed on an intention to treat basis. Subgroup analysis was performed for predefined subgroups based on age and type of recruiting physician. Differences in dichotomous outcomes (between "recovered" patients and "not recovered" patients) were analysed using logistic regression techniques for repeated measurements (including measurements at 6 weeks and at 3, 6, 9, and 12 months) and expressed in odds ratios. Differences in continuous outcomes (pain scores and functional scores) were analysed with linear regression techniques for repeated measurements, which take the correlation of multiple measurements within one patient into account.

Possible prognostic variables (age, gender, BMI, duration of knee symptoms, presence of bilateral symptoms, educational level, work participation, sports participation, and recruiting physician) were tested for their prognostic value in univariate regression analyses. All analyses were adjusted for baseline values and for possible prognostic factors.

The influence of exercise therapy on each outcome was tested using a model that included prognostic variables with a $P$ value of 0.1 or less and baseline values for pain at rest, pain on activity, and function score. Although the sample size calculation was based on a one sided testing approach, for the convenience of the reader we chose to show the results for the more conservative two sided tests, which were statistically significant at a $\mathrm{P}$ value of 0.05 . For statistically significant dichotomous outcomes, the number needed to treat is given (defined as $1 /$ risk difference for the defined outcome). For continuous data, we report effect sizes (Cohen's $d$ ), which are defined as the difference in outcome between the groups divided by the standard deviation of the baseline scores for this outcome. ${ }^{20}$ Differences in the number of additional interventions used in both groups were tested with Chi square statistics at a significance level of $\mathrm{P}=0.05$.

Analyses were conducted with SPPS 12.0 (SPSS; Chicago, IL, USA) and SAS 8.2 (SAS Institute; Cary, NC, USA).

\section{RESULTS}

Between April 2005 and April 2007, 163 patients with patellofemoral pain syndrome were recruited by the participating GPs and sport physicians. Of these individuals, 16 patients did not meet the inclusion criteria, 10 withdrew consent after being informed more extensively, and six were excluded because their symptoms had diminished. Therefore, a total of 131 patients were enrolled in the study and randomly assigned to the intervention group or the control group.

Figure 1 shows the recruitment, inclusion, assignment, and subsequent follow-up of the study patients.

The exercise therapy group $(\mathrm{n}=65)$ and control group $(\mathrm{n}=66)$ had similar baseline characteristics (table 1). Almost twice as many women as men were included in the whole sample. Bilateral knee symptoms were 


\begin{tabular}{|c|c|c|c|}
\hline & $\begin{array}{l}\text { Exercise therapy } \\
\qquad(n=65)\end{array}$ & $\begin{array}{l}\text { Control } \\
(n=66)\end{array}$ & $\begin{array}{l}\text { Total } \\
(\mathrm{n}=131)\end{array}$ \\
\hline Age in years (mean (SD)) & $24.7(8.6)$ & $23.3(7.8)$ & $24.0(8.2)$ \\
\hline Age $\geq 18$ years $(\%)$ & 69.3 & 69.7 & 69.5 \\
\hline Male gender (\%) & 35.4 & 36.4 & 35.9 \\
\hline BMI (mean (SD)) & $23.2(3.9)$ & $23.0(3.4)$ & $23.1(3.6)$ \\
\hline \multicolumn{4}{|l|}{ Duration of symptoms } \\
\hline 2-6 months (\%) & 69.2 & 66.6 & 67.9 \\
\hline 6-24 months (\%) & 31.8 & 33.4 & 32.1 \\
\hline Bilateral knee symptoms (\%) & 55.4 & 65.2 & 60.3 \\
\hline \multicolumn{4}{|l|}{ Educational level } \\
\hline Low (elementary school, lower level high school) (\%) & 23.4 & 22.7 & 23.6 \\
\hline $\begin{array}{l}\text { Medium or high (upper level high school, vocational } \\
\text { college, university) (\%) }\end{array}$ & 76.6 & 77.3 & 76.4 \\
\hline \multicolumn{4}{|l|}{ Hours of work a week } \\
\hline None (\%) & 26.2 & 28.8 & 27.4 \\
\hline$\ll 25$ hrs (\%) & 35.4 & 36.4 & 35.9 \\
\hline$\geq 25$ hrs (\%) & 38.5 & 34.8 & 36.6 \\
\hline Sports participation (\%) & 75.4 & 75.8 & 75.6 \\
\hline \multicolumn{4}{|l|}{ Recruiting physician } \\
\hline General practitioner (\%) & 76.9 & 77.3 & 77.1 \\
\hline Sport physician (\%) & 23.1 & 22.7 & 22.9 \\
\hline Function score out of 100 (mean (SD)) & 64.4 (13.9) & $65.9(15.2)$ & $65.1(14.5)$ \\
\hline Pain at rest out of 10 (mean (SD)) & $4.14(2.3)$ & $4.03(2.3)$ & $4.08(2.3)$ \\
\hline Pain on activity out of 10 (mean (SD)) & $6.32(2.2)$ & $5.97(2.3)$ & $6.15(2.2)$ \\
\hline
\end{tabular}

BMI, body mass index; SD, standard deviation.

more common than unilateral symptoms, and the study population had a high level of sports participation.

\section{Primary outcome parameters}

Tables 2 and 3 show the primary outcome measurements (recovery, function scores, and pain) at baseline and at 3 and 12 months follow-up. Outcomes at 3 and 12 months were missing for some patients, but available data from other time points were included in the analyses. This approach meant that the number of patients was not always equal for the different outcome measures.

Both the intervention and control group had a lower pain score at 3 months follow-up than at baseline. The adjusted analysis at 3 months showed a significant difference in pain at rest $(-1.07,95 \% \mathrm{CI}-1.92$ to -0.22 ; $\mathrm{P}=0.01)$ and pain on activity $(-1.00,-1.91$ to -0.08 ; $\mathrm{P}=0.03)$ in favour of the exercise group. The function score was considerably higher in the exercise than in the control group (adjusted difference 4.92, 0.14 to $9.72 ; \mathrm{P}=0.04)$. Effect sizes for exercise therapy ranged from 0.47 (pain at rest) and 0.45 (pain on activity) to 0.34 (function).

There was no significant difference in self reported recovery, as defined by the outcome measurement "recovered," between the groups at 3 months. When we used the outcome measurement "improved" (that is, "fully recovered," "strongly recovered," or "slightly recovered"), however, we found that recovery at 3 months was significantly more likely in the exercise group than in the control group (81\% improved $v 53 \%$ improved; adjusted odds ratio $4.07,95 \%$ CI 1.86 to 8.90; number needed to treat 3.6).

Between 3 and 12 months, another eight patients were lost to follow-up (five in the intervention group and three in the control group; see fig 1). One person who was lost before the 3 month follow-up was located and available for follow-up in the 3-12 month period.

At the 12 month follow-up, further improvement on pain and function scores from baseline was noted for both groups. The adjusted differences in pain scores between the groups still showed a significant difference in favour of the exercise group (pain at rest $-1.29,95 \%$ $\mathrm{CI}-2.16$ to $-0.42 ; \mathrm{P}<0.01$ and pain on activity -1.19 , -2.22 to $-0.16 ; \mathrm{P}=0.02)$. The effect sizes for exercise therapy on pain were 0.56 and 0.54 , respectively. The difference in function scores at 12 months, however, did not reach statistical significance $(4.52,95 \%$ CI -0.73 to $9.76 ; \mathrm{P}=0.09)$. The different between the two groups in the proportion of patients reporting "recovery" at 12 months was not significant.

Additional analysis of the data excluding the participants who violated the protocol during the first 3 months of follow-up showed greater differences in the outcome parameters of pain and function at 3 and 12 months. The odds ratio for the outcome parameter "recovery" at 12 months increased from 1.60 to 2.10 (95\% CI 0.94 to $4.66 ; \mathrm{P}=0.07$ ).

\section{Subgroup analysis}

Tables 4 and 5 present data for the subgroup analysis by recruiting physician. Among patients recruited by a GP, those in the exercise group had significantly higher and clinically relevant differences on the pain and functional outcome parameters compared with the control group at both 3 and 12 months follow-up (effect size pain at rest $0.67(\mathrm{P}<0.01)$ at 3 months and 0.79 $(\mathrm{P}<0.01)$ at 12 months; effect size pain on activity $0.62(\mathrm{P}<0.01)$ and $0.65(\mathrm{P}=0.02)$; and effect size function $0.57(\mathrm{P}<0.01)$ and $0.55(\mathrm{P}<0.01))$. Among patients recruited by a sport physician, however, those in the exercise group did not show better outcomes than

Table $2 \mid$ Recovery at 3 and 12 months follow-up

\begin{tabular}{|c|c|c|c|c|c|c|c|c|}
\hline & \multicolumn{3}{|c|}{$\begin{array}{l}\text { Exercise therapy } \\
\qquad(n=65)\end{array}$} & \multicolumn{3}{|c|}{$\begin{array}{l}\text { Control } \\
(n=66)\end{array}$} & \multirow{2}{*}{$\begin{array}{l}\text { Adjusted odds } \\
\text { ratio }(95 \% \mathrm{Cl}) \text { at } \\
3 \text { months }\end{array}$} & \multirow{2}{*}{$\begin{array}{l}\text { Adjusted odds } \\
\text { ratio }(95 \% \mathrm{Cl}) \text { at } \\
12 \text { months }\end{array}$} \\
\hline & Baseline & 3 months (n/N (\%)) & 12 months (n/N (\%)) & Baseline & 3 months $(\mathrm{n} / \mathrm{N}(\%))$ & 12 months ( $\mathrm{n} / \mathrm{N}(\%))$ & & \\
\hline Recovered* & - & $26 / 62(41.9)$ & $36 / 58(62.1)$ & - & $21 / 60(35.0)$ & $30 / 59(50.8)$ & 1.34 (0.65 to 2.79$)$ & 1.60 (0.77 to 3.34$)$ \\
\hline
\end{tabular}

Frequencies are reported for those patients available at that time point. Adjusted odds ratios are reported for the total available in analysis.

*Recovered=fully or strongly recovered.

†Recovery was adjusted for duration of symptoms. 
Table 3 Function and pain scores at 3 and 12 months follow-up

\begin{tabular}{|c|c|c|c|c|c|c|c|c|}
\hline & \multicolumn{3}{|c|}{$\begin{array}{l}\text { Exercise therapy } \\
\qquad(n=65)\end{array}$} & \multicolumn{3}{|c|}{$\begin{array}{l}\text { Control } \\
(n=66)\end{array}$} & \multirow[b]{2}{*}{$\begin{array}{l}\text { Adjusted difference* } \\
(95 \% \mathrm{Cl}) \text { at } 3 \text { months }\end{array}$} & \multirow[b]{2}{*}{$\begin{array}{l}\text { Adjusted difference* } \\
(95 \% \mathrm{Cl}) \text { at } 12 \text { months }\end{array}$} \\
\hline & $\begin{array}{c}\text { Baseline } \\
\text { (mean (SD)) }\end{array}$ & $\begin{array}{l}3 \text { months } \\
\text { (mean (SD)) }\end{array}$ & $\begin{array}{l}12 \text { months } \\
\text { (mean (SD)) }\end{array}$ & $\begin{array}{c}\text { Baseline } \\
\text { (mean (SD)) }\end{array}$ & $\begin{array}{l}3 \text { months } \\
\text { (mean (SD)) }\end{array}$ & $\begin{array}{l}12 \text { months } \\
\text { (mean (SD)) }\end{array}$ & & \\
\hline Function score $(0-100)$ & $64.4(13.9)$ & $78.8(15.5)$ & $83.2(14.8)$ & $65.9(15.2)$ & $74.9(17.6)$ & $79.8(17.5)$ & $4.92(0.14$ to 9.72$)$ & $4.52(-0.73$ to 9.76$)$ \\
\hline Pain at rest $(0-10)$ & $4.14(2.3)$ & $2.30(2.5)$ & $1.43(2.2)$ & $4.03(2.3)$ & $3.22(2.8)$ & $2.61(2.9)$ & $-1.07(-1.92$ to -0.22$)$ & $-1.29(-2.16$ to -0.42$)$ \\
\hline Pain on activity (0-10) & $6.32(2.2)$ & $3.81(2.9)$ & $2.57(2.9)$ & $5.97(2.3)$ & $4.60(3.0)$ & $3.54(3.38)$ & $-1.00(-1.91$ to -0.08$)$ & $-1.19(-2.22$ to -0.16$)$ \\
\hline
\end{tabular}

Mean scores are reported for those patients available at that time point. Adjusted differences are reported for the total available in analysis.

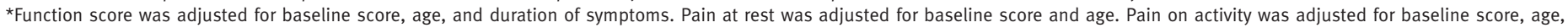
and gender. Positive adjusted differences for the function score, and negative difference for pain scores, are in favour of the exercise group.

those in the control group at either follow-up point Still no significant differences were found between the treatment and intervention groups for recovery at 3 and 12 months.

A further subgroup analysis was done on the basis of age. The effect estimates for recovery, pain, and function at 3 and 12 months for patients aged 14-17 years and for those aged 18 years or older were similar to those in the whole cohort. Because of lower power, there were no significant differences between the exercise therapy and control groups according to age, except for pain on activity at 3 months and pain at rest at 12 months in patients aged 18 years or older.

\section{Additional interventions}

There was no significant difference between the intervention group and the control group in the self reported total amount of additional interventions used (non-steroidal anti-inflammatory drugs (NSAIDs), bandages/braces, insoles, oral medication, and topical agents) during the first 3 months (that is, when participants in the intervention group were receiving the tailored exercise therapy). The use of oral NSAIDs and topical agents in the control group, however, was two to four times higher than in the intervention group $(\mathrm{P}=0.096$ and $\mathrm{P}=0.051$, respectively; fig 1).

Analysis of interventions used during the following 9 months (up to 12 months follow-up) showed similar disparities between the two groups in additional intervention use. The self reported use of NSAIDs and topical agents was about three times higher in the control group than in the intervention group $(\mathrm{P}=0.059$ and $\mathrm{P}=0.09$, respectively), whereas the use of supportive aids (bandages/braces) was about two times higher in the control group $(\mathrm{P}=0.09)$.

\section{DISCUSSION}

In patients with patellofemoral pain syndrome, exercise therapy produces better results regarding pain and function at 3 months and at 12 months than usual care.

We did not find a significant difference between the exercise therapy group and the control group in self reported "recovery" (that is, patients who designated themselves as "fully recovered" or "strongly recovered") at either 3 months or 12 months. Recovery at 3 months was significantly more likely in the exercise group than in the control group when we used the outcome measurement "improved" (that is, "fully recovered," "strongly recovered," or "slightly recovered"). After 12 months, nearly all patients had improved, and the difference between the groups was no longer significant.

We therefore conclude that, although exercise therapy is effective for improving pain and function, these benefits are not clearly reflected in patients' self reported recovery. Although perceived recovery is relevant as a clinical outcome, understanding what exactly comprises recovery from the patient's point of view is difficult.

We suspected that external factors might influence prognosis and possibly also effectiveness, so we stratified our analysis for age and type of recruiting physician. Clinically relevant and statistically significant effects of exercise on pain and function were found in patients recruited by the GP. This subgroup was relatively large $(n=101)$ and contributed considerably to the overall results. The group recruited by the sport

Table $4 \mid$ Recovery at 3 and 12 months follow-up for patients recruited by a general practitioner compared with those recruited by a sport physician

\begin{tabular}{|c|c|c|c|c|c|c|c|c|}
\hline & \multicolumn{3}{|c|}{$\begin{array}{l}\text { Exercise therapy } \\
\qquad(n=50)\end{array}$} & \multicolumn{3}{|c|}{$\begin{array}{l}\text { Control } \\
(n=51)\end{array}$} & \multirow{2}{*}{$\begin{array}{l}\text { Adjusted odds } \\
\text { ratio }(95 \% \mathrm{Cl}) \text { at } \\
3 \text { months }\end{array}$} & \multirow{2}{*}{$\begin{array}{l}\text { Adjusted odds } \\
\text { ratio }(95 \% \mathrm{Cl}) \text { at } \\
12 \text { months }\end{array}$} \\
\hline & Baseline & $\begin{array}{l}3 \text { months } \\
(\mathrm{n} / \mathrm{N}(\%))\end{array}$ & $\begin{array}{l}12 \text { months } \\
(\mathrm{n} / \mathrm{N}(\%))\end{array}$ & Baseline & $\begin{array}{l}3 \text { months } \\
(\mathrm{n} / \mathrm{N}(\%))\end{array}$ & $\begin{array}{l}12 \text { months } \\
(\mathrm{n} / \mathrm{N}(\%))\end{array}$ & & \\
\hline \multicolumn{9}{|c|}{ Patients recruited by general practitioners } \\
\hline Recovered* & - & $22 / 48(45.8)$ & $28 / 44(63.4)$ & - & $14 / 46(30.4)$ & $22 / 45(48.8)$ & 2.10 (0.89 to 4.93$)$ & $1.95(0.82$ to 4.65$)$ \\
\hline \multicolumn{9}{|c|}{ Patients recruited by sport physicians } \\
\hline Recovered* & - & 4/14 (28.6) & $8 / 14(57.1)$ & - & $7 / 14(50.0)$ & $8 / 14(57.1)$ & 0.39 (0.08 to 1.83$)$ & $0.97(0.22$ to 4.25$)$ \\
\hline
\end{tabular}

Frequencies are reported for those patients available at that time point. Adjusted odds ratios are reported for the total available in analysis. *Recovered=fully or strongly recovered.

†Recovery was adjusted for duration of symptoms. 
Table 5 Function and pain scores at 3 and 12 months follow-up for patients recruited by a general practitioner compared with those recruited by a sport physician

\begin{tabular}{|c|c|c|c|c|c|c|c|c|}
\hline & \multicolumn{3}{|c|}{$\begin{array}{l}\text { Exercise therapy } \\
\qquad(n=50)\end{array}$} & \multicolumn{3}{|c|}{$\begin{array}{l}\text { Control } \\
(n=51)\end{array}$} & \multirow[b]{2}{*}{$\begin{array}{l}\text { Adjusted difference* } \\
(95 \% \mathrm{Cl}) \text { at } 3 \text { months }\end{array}$} & \multirow[b]{2}{*}{$\begin{array}{l}\text { Adjusted difference* } \\
(95 \% \mathrm{Cl}) \text { at } 12 \text { months }\end{array}$} \\
\hline & $\begin{array}{c}\text { Baseline } \\
\text { (mean (SD)) }\end{array}$ & $\begin{array}{l}3 \text { months } \\
\text { (mean (SD)) }\end{array}$ & $\begin{array}{l}12 \text { months } \\
\text { (mean (SD)) }\end{array}$ & $\begin{array}{c}\text { Baseline } \\
\text { (mean (SD)) }\end{array}$ & $\begin{array}{c}3 \text { months } \\
\text { (mean (SD)) }\end{array}$ & $\begin{array}{l}12 \text { months } \\
\text { (mean (SD)) }\end{array}$ & & \\
\hline \multicolumn{9}{|c|}{ Patients recruited by general practitioners } \\
\hline Function score $(0-100)$ & $63.9(14.0)$ & $79.2(15.5)$ & $84.7(13.2)$ & $66.6(14.8)$ & $73.4(17.8)$ & $78.6(18.3)$ & 8.23 (3.18 to 13.28$)$ & $7.90(2.20$ to 13.60$)$ \\
\hline Pain at rest $(0-10)$ & $4.50(2.3)$ & $2.22(2.3)$ & $1.23(2.0)$ & $4.18(2.4)$ & $3.50(2.8)$ & $2.82(3.1)$ & $-1.56(-2.52$ to -0.61$)$ & $-1.82(-2.82$ to -0.82$)$ \\
\hline Pain on activity $(0-10)$ & $6.44(2.2)$ & $3.78(2.8)$ & $2.45(2.6)$ & $5.98(2.4)$ & $4.91(2.9)$ & $3.64(3.5)$ & $-1.42(-0.39$ to -2.45$)$ & $-1.49(-0.29$ to -2.69$)$ \\
\hline \multicolumn{9}{|c|}{ Patients recruited by sports physicians } \\
\hline Function score $(0-100)$ & $65.8(14.0)$ & $77.5(16.4)$ & $78.4(18.6)$ & $63.6(16.6)$ & $79.6(16.7)$ & $83.9(14.7)$ & $-4.11(-15.54$ to 7.33$)$ & $-5.31(-17.24$ to 6.62$)$ \\
\hline Pain at rest $(0-10)$ & $2.93(2.1)$ & $2.57(3.3)$ & $2.07(2.7)$ & $3.53(1.9)$ & $2.29(2.5)$ & $1.93(2.2)$ & $0.77(-1.15$ to 2.69$)$ & $0.56(-1.15$ to 2.28$)$ \\
\hline Pain on activity $(0-10)$ & $5.93(1.9)$ & $3.93(3.4)$ & $2.93(3.5)$ & $5.93(2.0)$ & $3.57(3.0)$ & $3.21(3.0)$ & $0.41(-1.83$ to 2.64$)$ & $-0.21(-2.49$ to 2.06$)$ \\
\hline
\end{tabular}

Mean scores are reported for those patients available at that time point. Adjusted differences are reported for the total available in analysis.

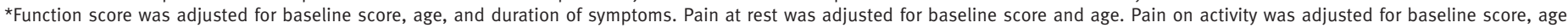
and gender. Positive adjusted differences for the function score, and negative difference for pain scores, are in favour of the exercise group.

physician was small $(\mathrm{n}=30)$, however, and did not show any effect of exercise therapy compared with usual care. The confidence intervals for this analysis were wide, so coincidental findings owing to the small numbers of patients recruited by sport physicians cannot be excluded. There were no possible explanatory differences in baseline characteristics (including frequency and duration of symptoms, sports participation, and BMI) between patients recruited by a GP and those recruited by a sport physician.

There was no difference between the exercise therapy group and the control group in the number of additional interventions used during the first 3 months of the study, although there was a two-fold to three-fold higher use of NSAIDs and a four times higher use of topical agents in the control group. These figures remained stable during the course of one year. These additional interventions might have influenced the outcome measurements. The use of additional interventions was higher in the control group, however, implying that differences in outcome measurements between the groups are more likely to be underestimated than overestimated. Although not significant, the higher use of additional interventions in the control group may indicate a trend towards greater use of self supportive means by patients with patellofemoral pain syndrome not referred to an exercise programme. When considered alongside the better outcome on pain scores in the exercise group, the data indicate that the control group had an objective need to use pain medication.

\section{Comparison with other studies}

Various studies have evaluated the efficacy of exercise therapy for patellofemoral pain syndrome. Early studies without a control group indicated that rehabilitation including exercise therapy could be beneficial for patients with patellofemoral pain syndrome..$^{21} 22$

Witvrouw et al studied the effect of open compared with closed chain exercises in patients with patellofemoral pain syndrome. ${ }^{2324}$ Both types of exercise led to an improvement in pain scores and an increase in strength of the quadriceps and hamstrings. The authors stated that, as a result of their study, they would use both open and closed kinetic chain exercises in the non-operative treatment protocol for patients with patellofemoral pain. ${ }^{2324}$ Given these findings, both isometric/concentric and eccentric exercises were used in the present study. Patients were also allowed to practise in an open and closed kinetic chain position.

Various other studies have evaluated the efficacy of exercise therapy for patellofemoral pain syndrome. Systematic reviews, however, have reported that most of these studies are of poor methodological quality in that they lack randomisation, a control group, or clearly defined outcome parameters. ${ }^{1225}$

To our knowledge, six randomised studies, including our own study, have compared exercise therapy with non-exercise therapy. One low quality study found that a special brace designed to provide progressive resistance exercise during activities of daily living improved function and pain, ${ }^{26}$ whereas another study found no significant differences in outcomes between patients on a home exercise programme and those with a patellar brace. ${ }^{27}$

Additionally, three trials studied the effects of physiotherapy (including exercise therapy) compared with other treatment or placebo treatment. ${ }^{192829}$ The small study by Crossley et al compared the effect of a 6 week programme of exercise therapy plus taping, mobilisation techniques, and biofeedback with a placebo therapy (sham ultrasound, placebo taping, and application of non-therapeutic gel) ${ }^{28}$ After 6 weeks, the multimodal physiotherapy group showed a significant decrease in worst pain, usual pain, and anterior knee pain compared with the placebo group. The recent study by Collins et al compared the use of insoles (flat or prefabricated) with 6 weeks of multimodal physiotherapy (including exercises). ${ }^{29}$ At 6,12 , and 52 weeks, no differences were found between groups regarding pain, function, and recovery.

These studies, however, do not answer the question of whether the effect of supervised exercises is additive to usual care, as was tested in our study. In the small 


\section{WHAT IS ALREADY KNOWN ON THIS TOPIC}

Both exercise therapy and a "wait and see" approach are advocated in patients with patellofemoral pain syndrome

There is only limited evidence for the effectiveness of exercise therapy with respect to pain outcomes and conflicting evidence with respect to functional outcomes

\section{WHAT THIS STUDY ADDS}

Supervised exercise therapy for patients with patellofemoral pain syndrome is more effective than usual care with respect to pain at rest, pain on activity, and knee function

Supervised exercise therapy has no significant effect on self reported recovery

study by Clark et al, ${ }^{19}$ exercise, taping, and education were compared with exercise and education, taping and education, and education alone. Exercise was not significantly better than non-exercise for pain and function after 3 months. At 12 months, however, pain scores were significantly better for the exercise group. A high proportion of patients (approximately 40\%) was lost to follow-up at 12 months in this study though, which could have influenced these results.

\section{Strengths and limitations of study}

A methodological problem that cannot be solved in randomised controlled trials of exercise therapy is that patients in the intervention group cannot be blinded for the exercise therapy and, therefore, may be biased for positive outcome (placebo effect). To overcome this problem in part, a blinded external observer could be used to provide objective and observational measures of functional outcomes. Some intervention studies on exercise therapy in patellofemoral pain syndrome have used quantifiable measures for muscle strength as an outcome. However, several studies have shown that the relationship between increase in muscle strength and clinical outcome is inconsistent. ${ }^{30-32}$ In addition, these studies clearly illustrate the difficulty of interpreting the effect of therapy using muscle strength as an outcome measure for knee function. Therefore, as no validated objective outcome measures for patellofemoral pain syndrome are currently available, the use of validated subjective outcome measures seems appropriate.

Along with the observed effect of exercise therapy, the role of supervision and attention of a physical therapist as well as the use of an exercise diary may have influenced the outcome in the intervention group. The attention from the physical therapist is an integral part of the supervised exercise therapy intervention. On the other hand, the use of an exercise diary in the intervention group to assess compliance may have caused a bias owing to awareness of being involved in a study (Hawthorne effect). ${ }^{33}$

In addition, our control group was allowed to do single isometric quadriceps contractions and is, therefore, not a real non-exercise group. This resembles the usual care ("wait and see" approach) prescribed by GPs in the Netherlands according to national guidelines. ${ }^{9}$ We thus studied the additional value of supervised exercise therapy but not the effect of doing exercises, which might have diminished the contrast between the groups. Although we noted that eight patients in the control group received physical therapy (that is, they violated the protocol), we do not know to what extent this physical therapy resembled the standardised supervised exercise therapy in the intervention group. Therefore, an analysis by protocol by comparing physical therapy with no physical therapy does not seem appropriate.

The differences between the intervention group and the control group were further diminished by violation of the protocol by 14 people: 6 people in the intervention group did not visit the physical therapist and subsequently did not receive the supervised exercise therapy, and eight people in the control group visited a therapist and received physical therapy although they were instructed not to. Additional analysis of the data excluding the participants who violated the protocol during the first 3 months of follow-up showed greater differences in the outcome parameters on pain and function at 3 and 12 months. This change indicates that the effects of exercise therapy may indeed be even higher than those reported in our primary analysis.

A final remark can be made about the diagnosis of patellofemoral pain syndrome. Patients were recruited by GPs and sport physicians who were offered a set of inclusion and exclusion criteria, some of which were related to physical examination. The researchers did not supervise the physicians in their judgment of diagnosis. Nevertheless, we think that this approach reflects common practice and therefore increased the clinical applicability of our results.

\section{Conclusions}

This study provides evidence that supervised exercise therapy for patellofemoral pain syndrome in general practice is more effective than usual care" for the outcome parameters pain at rest, pain on activity, and function at 3 and 12 months. However, supervised exercise therapy had no effect on perceived recovery. Further research should aim to elucidate the mechanisms whereby exercise therapy results in better outcome.

Contributors: All authors except SPW were involved in the study design MvM, RvL, and SMB-Z coordinated the study and were responsible for the data collection. The statistical analyses were prepared and executed by RVL, MvM, SPW, SMB-Z, and BWK. All authors contributed to the interpretation of the analyses and writing the article. SMB-Z is the guarantor

Funding: This study was supported by ZON-MW (The Netherlands organisation for health research and development)

Competing interests: None declared.

Data sharing: Technical appendix, statistical code, and dataset available from the corresponding author at m.vanmiddelkoop@erasmusmc.nl.

1 Thomee R, Augustsson J, Karlsson J. Patellofemoral pain syndrome: a review of current issues. Sports Med 1999;28:245-62.

2 Sanchis-Alfonso V. Anterior knee pain and patellar instability. Springer Verlag, 2006.

3 Witvrouw E, Werner S, Mikkelsen C, Van Tiggelen D, Vanden Berghe L, Cerulli G. Clinical classification of patellofemoral pain syndrome: guidelines for non-operative treatment. Knee Surg Sports Traumatol Arthrosc 2005;13:122-30. 
$4 \quad$ Van der Velden J. Een nationale studie naar ziekten en verrichtingen in de huisartspraktijk. Basisrapport: morbiditeit in de huisartspraktijk. Utrecht: NIVEL, 1991.

5 Taunton JE, Ryan MB, Clement DB, McKenzie DC, Lloyd-Smith DR, Zumbo BD. A retrospective case-control analysis of 2002 running injuries. Br J Sports Med 2002;36:95-101.

6 Dixit S, DiFiori JP, Burton M, Mines B. Management of patellofemoral pain syndrome. Am Fam Physician 2007;75:194-202.

7 Den Hertog PC, van Kampen LTB, Ooijendijk WTM, Schmikli SL, Schoots W, Vriend I. Ongevallen en Bewegen in Nederland: Kerncijfers 2000-2001 voor beleid en onderzoek. Stichting Consument en Veiligheid, 2003.

8 Nimon G, Murray D, Sandow M, Goodfellow J. Natural history of anterior knee pain: a 14- to 20-year follow-up of nonoperative management. J Pediatr Orthop 1998;18:118-22.

9 Cirkel JW, Klaassen WRC, Kunst JA, Aarns TEM, Plag EC, Goudswaard AN, et al. Niet-Traumatische knieproblemen bij kinderen en adolescenten; NHG-Standaard. Huisarts Wet 1998;41:246-51.

10 Cutbill JW, Ladly KO, Bray RC, Thorne P, Verhoef M. Anterior knee pain: a review. Clin J Sport Med 1997;7:40-5.

11 McConnell J. The physical therapist's approach to patellofemoral disorders. Clin Sports Med 2002;21:363-87.

12 Heintjes E, Berger MY, Bierma-Zeinstra SM, Bernsen RM, Verhaar JA Koes BW. Exercise therapy for patellofemoral pain syndrome. Cochrane Database Syst Rev 2003(4):CD003472.

13 Van Linschoten R, van Middelkoop M, Berger MY, Heintjes EM, Koopmanschap MA, Verhaar JA, et al. The PEX study-exercise therapy for patellofemoral pain syndrome: design of a randomized clinical trial in general practice and sports medicine [ISRCTN83938749]. BMC Musculoskelet Disord 2006;7:31.

14 Malanga GA, Andrus S, Nadler SF, McLean J. Physical examination of the knee: a review of the original test description and scientific validity of common orthopedic tests. Arch Phys Med Rehabil 2003;84:592-603.

15 Nijs J, Van Geel C, Van der auwera C, Van de Velde B. Diagnostic value of five clinical tests in patellofemoral pain syndrome. Man Ther 2006;11:69-77.

16 Kujala UM, Jaakkola LH, Koskinen SK, Taimela S, Hurme M, Nelimarkka O. Scoring of patellofemoral disorders. Arthroscopy 1993;9:159-63.

17 Downie WW, Leatham PA, Rhind VM, Wright V, Branco JA, Anderson JA. Studies with pain rating scales. Ann Rheum Dis 1978;37:378-81.

18 Crossley KM, Bennell KL, Cowan SM, Green S. Analysis of outcome measures for persons with patellofemoral pain: which are reliable and valid? Arch Phys Med Rehabil 2004;85:815-22.
19 Clark DI, Downing N, Mitchell J, Coulson L, Syzpryt EP, Doherty M. Physiotherapy for anterior knee pain: a randomised controlled trial. Ann Rheum Dis 2000;59:700-4.

20 Hartung J, Knapp G, Sinha BK. Statistical meta-analysis with application. Wiley, 2008.

21 Dehaven KE, Dolan WA, Mayer PJ. Chondromalacia patellae in athletes. Clinical presentation and conservative management. Am J Sports Med 1979;7:5-11.

22 Whitelaw GP Jr, Rullo DJ, Markowitz HD, Marandola MS, DeWaele MJ. A conservative approach to anterior knee pain. Clin Orthop Relat Res 1989:234-7.

23 Witvrouw E, Lysens R, Bellemans J, Peers K, Vanderstraeten G. Open versus closed kinetic chain exercises for patellofemoral pain. A prospective, randomized study. Am / Sports Med 2000;28:687-94.

24 Witvrouw E, Danneels L, Van Tiggelen D, Willems TM, Cambier D. Open versus closed kinetic chain exercises in patellofemoral pain: 5-year prospective randomized study. Am / Sports Med 2004;32:1122-30.

25 Bizzini M, Childs JD, Piva SR, Delitto A. Systematic review of the quality of randomized controlled trials for patellofemoral pain syndrome. J Orthop Sports Phys Ther 2003;33:4-20.

26 Timm KE. Randomized controlled trial of Protonics on patellar pain, position, and function. Med Sci Sports Exerc 1998;30:665-70.

27 Lun VM, Wiley JP, Meeuwisse WH, Yanagawa TL. Effectiveness of patellar bracing for treatment of patellofemoral pain syndrome. Clin J Sport Med 2005;15:235-40.

28 Crossley K, Bennell K, Green S, Cowan S, McConnell J. Physical therapy for patellofemoral pain: a randomized, double-blinded, placebo-controlled trial. Am J Sports Med 2002;30:857-65.

29 Collins N, Crossley K, Beller E, Darnell R, McPoil T, Vicenzino B. Foot orthoses and physiotherapy in the treatment of patellofemoral pain syndrome: randomised clinical trial. BMJ 2008;337:a1735.

30 Gobelet C, Frey M, Bonard A. Muscle training techniques and retropatellar chondropathy. Rev Rhum Mal Osteoartic 1992;59:23-7.

31 Stiene HA, Brosky T, Reinking MF, Nyland J, Mason MB. A comparison of closed kinetic chain and isokinetic joint isolation exercise in patients with patellofemoral dysfunction. J Orthop Sports Phys Ther 1996;24:136-41.

32 Dursun N, Dursun E, Kilic Z. Electromyographic biofeedbackcontrolled exercise versus conservative care for patellofemoral pain syndrome. Arch Phys Med Rehabil 2001;82:1692-5.

33 Mayo E. Hawthorne and the Western Electric Company: the social problems of an industrial civilisation. Routledge, 1949.

Accepted: 21 April 2009 